\section{Biomarker zur Vorhersage des Chemotherapie-Erfolgs?}

Forscher haben einen potentiellen Biomarker zur Vorhersage des Erfolgs einer Chemotherapie bei Darmkrebs identifiziert. Sie fanden heraus, dass eine bestimmte Modifikation des Gens TFAP2E damit zusammenhängt, ob ein Patient auf eine Chemotherapie anspricht.

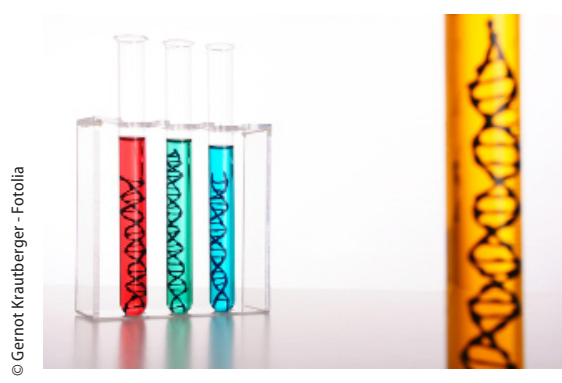

Wie die Ergebnisse einer Studie mit 200 Teilnehmern zeigten, bedingt die Methylierung des Gens TFAP2E ob ein Patient auf eine Chemotherapie anspricht oder nicht. Bei der Methylierung heftet ein Enzym CH3-Gruppen an das Cytosin der DNA und beeinflusst so die Aktivität von Genen. Die Forscher wiesen nach, dass Patienten mit einem besonders stark methylierten TFAP2E-Gen resistent gegen das Chemotherapeutikum 5-Fluorouracil waren. Dieses Ergebnis war unabhängig davon, ob die Patienten gleichzeitig eine Bestrahlungstherapie erhielten oder nicht.

Die Hypermethylierung des TFAP2E-Gens bewirkt, dass das Gen DKK4 vermehrt abgelesen wird. DKK4 ist Teil eines Signalwegs, der Informationen von der Zelloberfläche ins Zellinnere leitet und vermutlich eine Rolle bei der Entstehung von Darmkrebs spielt. Das Forscherteam möchte in Zukunft die Rolle der beiden Gene für die Darmkrebstherapie weiter erforschen und testen, ob sich aus der Studie ein Biomarker für den Einsatz in der Klinik ableiten lässt.

Literatur: Ebert M, Tänzer M, Balluff B et al. (2012) TFAP2E-DKK4 and Chemoresistance in Colorectal Cance. NEJM 366: 44-53 der EGFR-Protein-Expression betrachtet: Es wurde sowohl eine positive Korrelation zwischen EGFR-Expression und Ansprechen auf TKI gesehen [27] wie auch eine negative Korrelation [19]. Insgesamt weisen die Ergebnisse eher auf die bekannten Schwierigkeiten in der Quantifizierung von Proteinexpression hin, als dass sie Auskunft über die prognostische oder prädiktive Aussage der EGFR-Expression geben.

\section{KRAS-Mutation}

Neben den aktivierenden Mutationen, die EGFR selbst betreffen, können auch Proteine mutiert sein, die das am EGFR empfangene Signal zum Zellkern weiter leiten. Am häufigsten von aktivierenden Mutationen betroffen ist hier KRAS. KRAS-Mutationen, die die Codons 12, 13 und 61 betreffen, sind vor allen Dingen beim Kolorektalkarzinom bekannt. Hier vermitteln sie Resistenz gegen die Therapie mit Anti-EGFR-Antikörpern, z. B. Cetuximab [25], da sie Proliferationssignale unabhängig vom EGFR-Status in die Zelle abgeben.

Auch beim Lungenkarzinom ist KRAS mit einer Frequenz von etwa 22\% mutiert ([21], Abb. 2). Diese Patienten haben generell eine schlechtere Prognose ([13]; Metaanalyse von 53 Studien). Die prädiktive Bedeutung des Mutationsstatus ist nicht ganz so eindeutig geklärt. Insgesamt muss man aber davon ausgehen, dass zumindest die TKIs bei Patienten mit KRAS-Mutationen schlecht ansprechen [27].

Cetuximab. Schwieriger ist die Frage zu klären, ob auch Cetuximab beim NSCLC mit KRAS-Mutation als Therapieoption ausgeschlossen werden sollte, ebenso wie es beim Kolorektalkarzinom mit KRASMutation nicht indiziert ist. Die FLEXStudie [20], die den KRAS-MutationsStatus nicht berücksichtigt, kam zu dem Ergebnis, dass die Gabe von Cetuximab für Patienten mit NSCLC keinen signifikanten Überlebensvorteil bedeutet. Offen bleibt, ob sich das Ergebnis ändern würde, wenn die Patienten mit KRASMutation, wie beim Kolorektalkarzi- nom, von der Studie ausgeschlossen würden [20].

\section{BRAF-Mutation}

Gleich anschließend an KRAS folgt BRAF im EGFR-Signalweg. BRAF-Mutationen sind vor allen Dingen interessant, weil hier bereits klinisch erprobte oder in der Erprobung befindliche Inhibitoren vorliegen (Sorafenib, Vemurafenib, Selumetinib). Besonders Vemurafenib, ein spezifischer Inhibitor der BRAFV6ooE-Mutation, hat in der Therapie des metastasierten Melanoms schon beeindruckende Erfolge gezeigt [2]. Leider sind BRAF-Mutationen beim NSCLC seltener als bei anderen soliden Tumoren: Sie kommen nur mit einer Frequenz von etwa $3 \%$ vor [18]. Aufgrund der spärlichen Datenlage lassen sich bislang keine Aussagen über das prädiktive Potenzial von BRAF-Mutationen machen.

\section{» EGFR-Mutationen und KRAS- oder BRAF-Mutationen schließen}

\section{sich aus «}

EGFR-Mutationen und KRAS- oder BRAF-Mutationen schließen sich aus, d. h., wenn eine KRAS- oder BRAF-Mutation nachgewiesen wurde, kann man davon ausgehen, dass eine positiv-prädiktive EGFR-Mutation nicht vorliegt [10]. Dies ist auch im Rahmen der Kostenersparnis bei der Mutationstestung relevant.

\section{EML4-ALK-Translokation}

Eine weitere Mutation, die den KRASBRAF-Signalweg aktiviert, ist die EML4ALK-Translokation. In dem entstehenden Fusionsprotein ist der N-terminale Bereich von EML4 („,echinoderm microtubule-associated protein-like 4 “) mit der Kinasedomäne von ALK (, anaplastic lymphoma kinase") fusioniert, wodurch die ALK-Kinase aktiviert wird. EML4ALK-Translokationen beim NSCLC sind selten und werden nur bei 3-7\% aller Patienten nachgewiesen [6]. Trotzdem gibt es für sie nur 4 Jahre nach ihrer Erstbeschreibung bereits eine spezifische Therapie: Vor kurzem wurde Crizotinib von 